\title{
Synthetic Vision CFIT Experiments for GA and Commercial Aircraft: "A Picture Is Worth A Thousand Lives"
}

\author{
Lawrence J. Prinzel III, Monica F. Hughes, Jarvis J. Arthur III, Lynda J. Kramer, \\ Louis J. Glaab, Randy E. Bailey, Russell V. Parrish, Michael D. Uenking \\ NASA Langley Research Center, Hampton, VA
}

\begin{abstract}
Because restricted visibility has been implicated in the majority of commercial and general aviation accidents, solutions will need to focus on how to enhance safety during instrument meteorological conditions (IMC). The NASA Synthetic Vision Systems (SVS) project is developing technologies to help achieve these goals through the synthetic presentation of how the outside world would look to the pilot if vision were not reduced. The potential safety outcome would be a significant reduction in several accident categories, such as controlled-flight-into-terrain (CFIT), that have restricted visibility as a causal factor. The paper describes two experiments that demonstrated the efficacy of synthetic vision technology to prevent CFIT accidents for both general aviation and commercial aircraft.
\end{abstract}

\section{INTRODUCTION}

The Synthetic Vision Systems (SVS) element of the National Aeronautics and Space Administration's (NASA) Aviation Safety Program (AvSP) is striving to eliminate poor visibility as a causal factor in aircraft accidents as well as enhance operational capabilities of all aircraft. To accomplish these safety and situation awareness improvements, the SVS concept is designed to provide a clear view of the world ahead through the display of computer generated imagery derived from an onboard database of terrain, obstacle, and airport information and enhanced sensor technologies. NASA research conducted to date has successfully evaluated the safety benefits, but only during nominal, restricted visibility operations (e.g., Glaab \& Takallu, 2002; Prinzel et al., 2002; Prinzel et al., in press). Although the research has consistently shown the advantage of synthetic vision compared to traditional instruments for complex approaches to terrain- (EGE, ROA, AVL) or operationalchallenged airports (DFW), the true safety value of SVS would be to reduce or eliminate off-nominal situations that pose significant safety risks such as prevention of CFIT.

The paper describes the results of two experiments that were designed to evaluate the efficacy of synthetic vision for CFIT prevention. Experiment I focused on general aviation and introduced an inadvertent IMC scenario with an altimeter error that placed the low-hour VFR pilot in close proximity to threatening terrain. Experiment II focused on commercial air transport pilots and introduced a lateral path error in flight management system guidance that brought the aircraft into close proximity with terrain during a go-around procedure. A significant amount of data was collected during these two experiments, but only the results relevant to CFIT prevention are discussed.

\section{EXPERIMENT I}

\section{Participants}

Fourteen low-hour VFR (> 400 hours) pilots served as participants for Experiment I. None of the pilots had received any instrument training except for limited exposure during their private pilot license training. An additional six instrument pilots with less than 1000 hours, four professional test pilots, and three Capstone-II project pilots also participated $(\mathrm{N}=27)$.

\section{Display Concepts}

Although all participants flew a baseline concept (Figure 1) during the nominal trials, no data was collected on the baseline display during the CFIT trials because pilot study data revealed that even professional pilots were unable to avoid the terrain. Therefore, the inadvertent IMC anomaly scenario was designed to show that an otherwise unavoidable CFIT situation could be prevented with synthetic vision technology. Three SVS texturing concepts were evaluated: Constant Color (CC), Elevation-Based Generic (EBG), and Photo-Realistic (PR). CC replicates an industry concept that the FAA has certified under the Capstone-II program. The EBG concept uses shades of green with darker shades representing higher terrain. Finally, the PR concept was derived from 4-meter satellite imagery data. The display concepts were combined with 1,3 , or 30 arc-sec digital elevation models (DEM). A 500 x $500 \mathrm{ft}$ grid fishnet was also evaluated as secondary texturing concept.

Simulation Facility

The simulation equipment consisted of a modified Precision Flight Control PC-based Aviation Training Device (PCATD) hardware, and ELITE C-172R IFR (v.6.2) software. A 6" SVS-PFD and a UPSAT MX-20 MFD were added to the PCATD hardware. A Silicon Graphics Zx10 PC and a 3Dlab WildCat 4210 graphics card were used for synthetic vision display and out-the-window scene.

\section{Experimental Matrix}

A usability study was conducted that tested all 18-display concepts and the results were used to reduce the experimental matrix to 10 display concepts. Because of the uneven number of pilots available for data collection, these display concepts were then randomly drawn for each pilot participant to fly during the CFIT scenario. Details of the method and usability study are reported in Hughes and Takallu (2002).

\section{CFIT Scenario}

The CFIT scenario consisted of a situation in which the pilot incorrectly set the barometric altimeter to read 1500 feet higher than actual altitude. Such an error would affect both the altimeter and MX-20 readings, but not the SVS concept because the system receives inputs from a Wide Area Augmentation System (WAAS) receiver. Pilots had flown 34 experimental runs prior to the CFIT scenario (35 total). The CFIT scenario resembled 11 of the previous 34 trials that began straight-and-level at $6500 \mathrm{ft}$ MSL (4000 ft AGL) with instructions to make a left-bank turn and descend after two minutes to $5000 \mathrm{ft}$ MSL (1000 ft AGL) over rising terrain. The scenario began in VMC with visibility deteriorating to IMC within one-minute elapsed time. The CFIT scenario started at $5000 \mathrm{ft}$ MSL, but the altimeter showed $6500 \mathrm{ft}$ MSL. Therefore, the instruction to reduce altitude by $1500 \mathrm{ft}$ in effect descended the aircraft to $-500 \mathrm{ft}$ below the mountain peaks directly in front of the aircraft. 


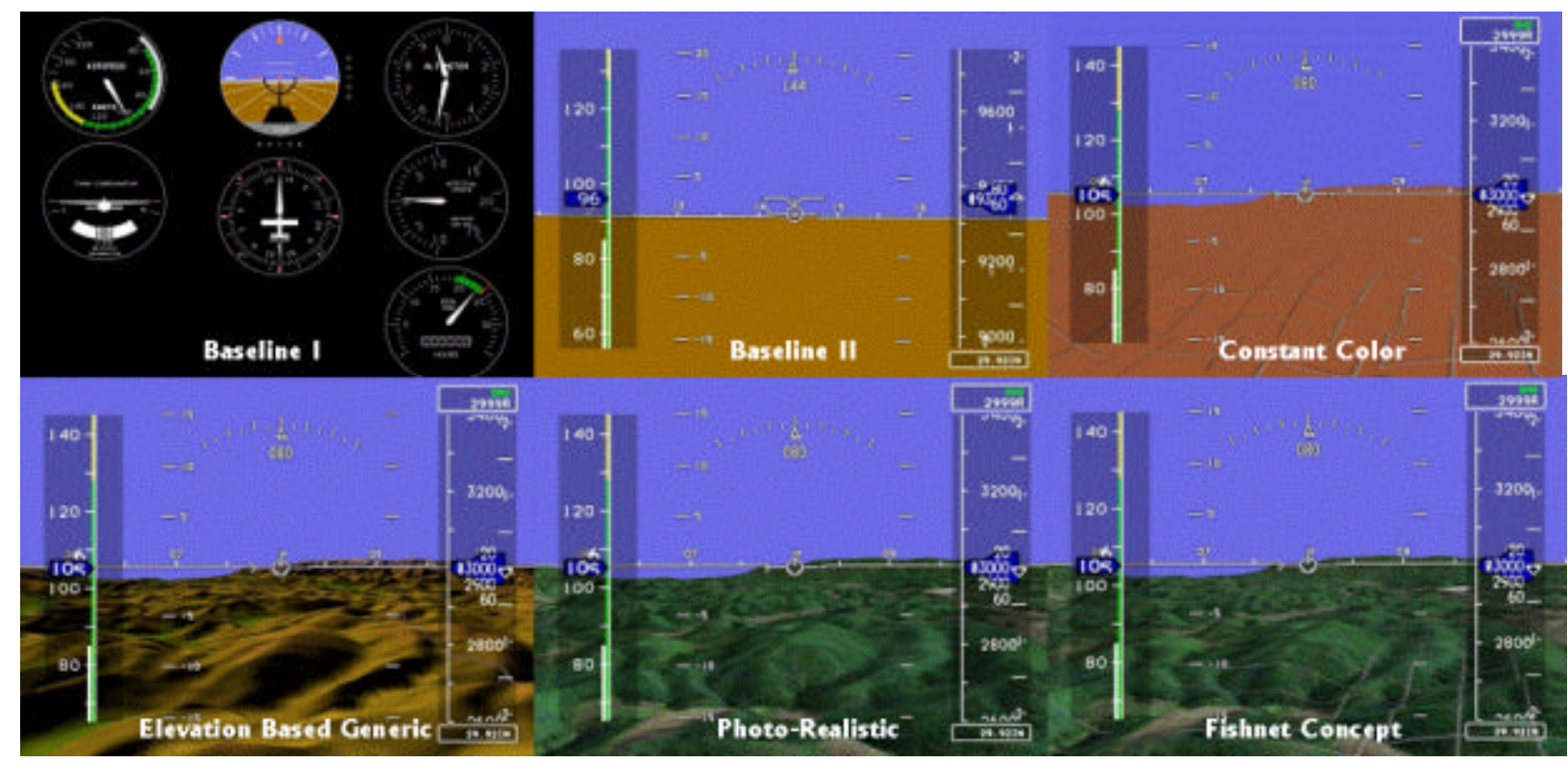

Figure 1. Display Concepts For Experiment I

Results

\section{CFIT Event Detection}

None (0/13) of the IFR and professional pilots and only $15 \%(2 / 14)$ of the VFR pilots experienced a CFIT. One of the pilots had significant difficulty flying the aircraft throughout the entire experimental session and analysis showed performance to be well outside practical pilot standards; therefore, the data may be viewed suspect. The other pilot, however, did experience a CFIT event and, during the semistructured interview, reported awareness that something was wrong but felt captured by the incorrect MX-20 reading and failed to crosscheck the instruments. Figure 2 presents average mean time-to-impact before detection and average AGL for CFIT scenario.
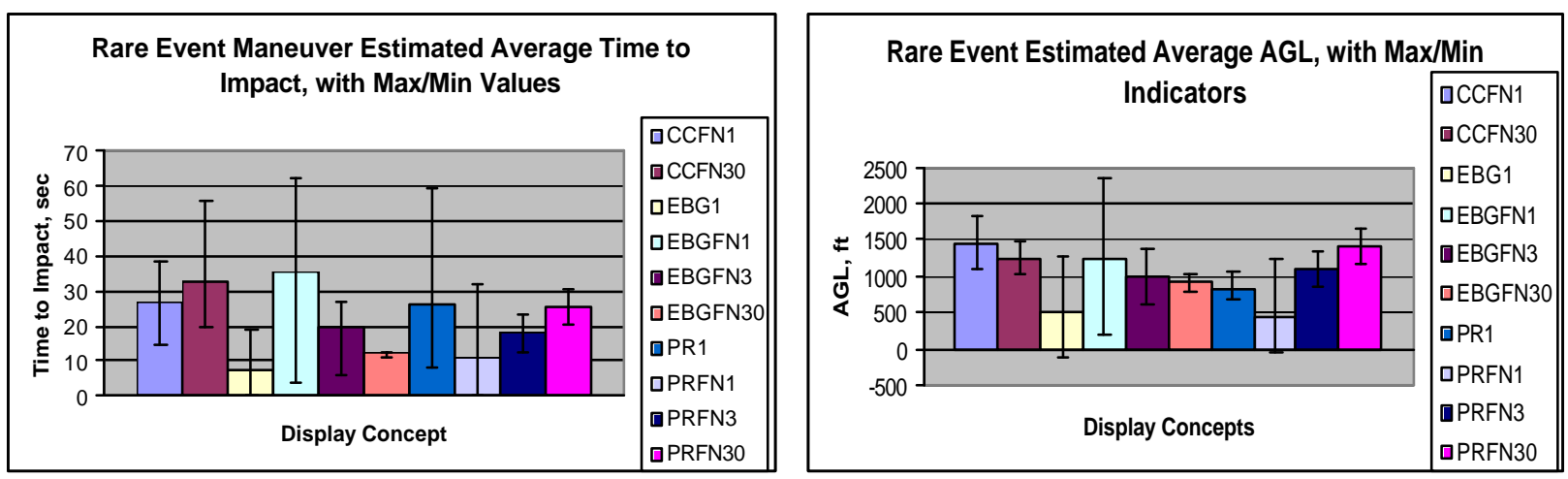

Physiological Response

Figure 2. Average Mean Time-To-Impact Before Detection and Average AGL Altitude

Physiological measures of heart rate (HR), skin temperature (ST), and electromyography (EMG) were collected 3.2 miles into the run. Although not significant ( $\mathrm{p}>.05$ ), Figure 3 shows an increase in physiological response that may reflect an awareness of the potential CFIT event (dotted line). ST is reversed scored and the figure depicts a decrease in ST pri or to event. Please note that a decrease in ST reflects an increase in physiological arousal.
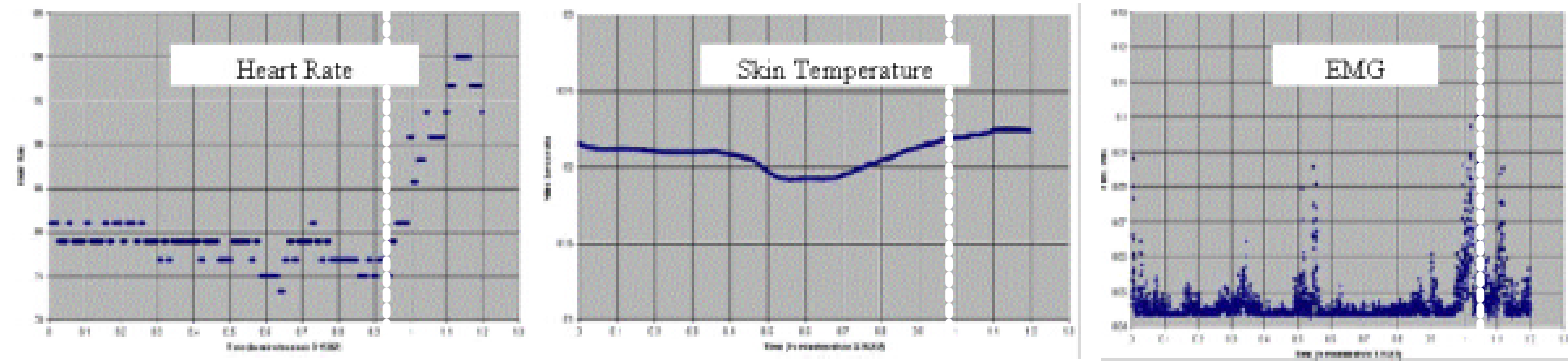

Figure 3. Example Psychophysiological Data During CFIT Scenario 


\section{EXPERIMENT II}

Pilot Participants

A total of 15 airline pilots and 1 NASA researcher participated in the experiment $(\mathrm{N}=16)$. All subjects had HUD experience and all airline pilots had current commercial licenses. The subjects had an average 20.2 years of flying experience with an average 8600 hours logged. The subjects were given a 30-minute briefing and a 2-hour training session. Pilots were instructed that their primary responsibility was terrain avoidance and to crosscheck all cockpit indications.

Simulation Facility

The experiment was conducted in the Visual Imaging Simulator for Transport Aircraft Systems (VISTAS) III part task simulator at NASA Langley Research Center. The single pilot fixed based simulator consists of a $144^{\circ}$ by $30^{\circ} \mathrm{OTW}$ scene, a simulated HUD, a large field HDD and pilot input controls. The OTW scene was used only during training. The pilot controls in the VISTAS III workstation are a left side arm controller, left/right throttle controls, rudder pedals, left/right toe brakes and a PC track ball for display related pilot inputs.

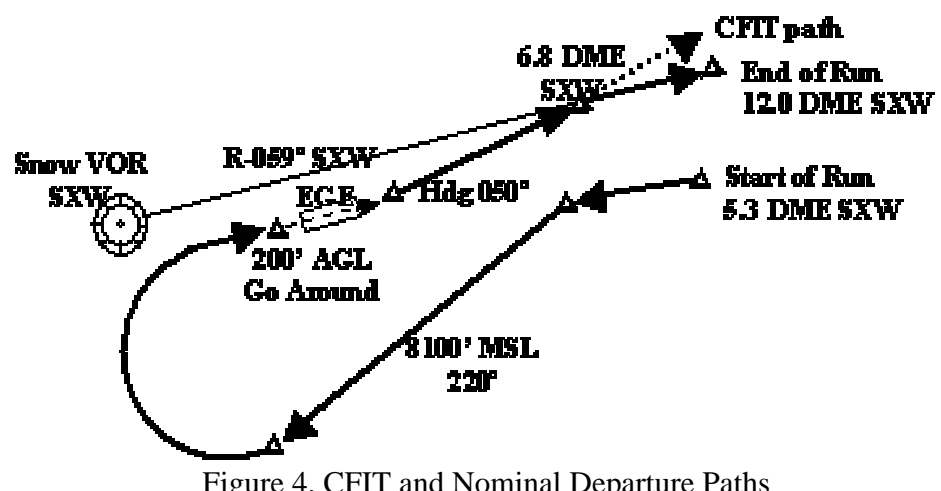

Evaluation Tasks

Pilots were asked to fly a circling approach to Eagle-Vail, CO (EGE) runway 07 under CAT IIIa and execute a go-around $200 \mathrm{ft}$ AGL and intercept the 059 radial from SNOW VOR (Figure 4). The aircraft model was a Boeing 757, and both the approach and departure speed target was 140 knots. All scenarios were flown with moderate turbulence. For the approach part of the task, auto throttles were enabled, flaps were set to 30 degrees and the landing gear was down. At $200 \mathrm{ft}$ AGL, a go around was executed and the climb gradient performance was degraded. The pilot raised the landing gear and the flaps were set to go-around configuration. The evaluation pilot was instructed to use speed-on-pitch to maintain 140 knots and follow the departure path that provided escape guidance through a "notch" between two mountain peaks. The run ended at the 12.0 DME point from SXW. For the CFIT scenario (run 22 of 22), the flight guidance was altered on the departure path (dotted path in Figure 4) and was the only difference between the CFIT scenario and the previous 21 data runs. A Terrain Awareness Warning System (TAWS) and Vertical Situation Display (VSD), however, were available on the navigation display for both baseline and SVS (Figure 5).

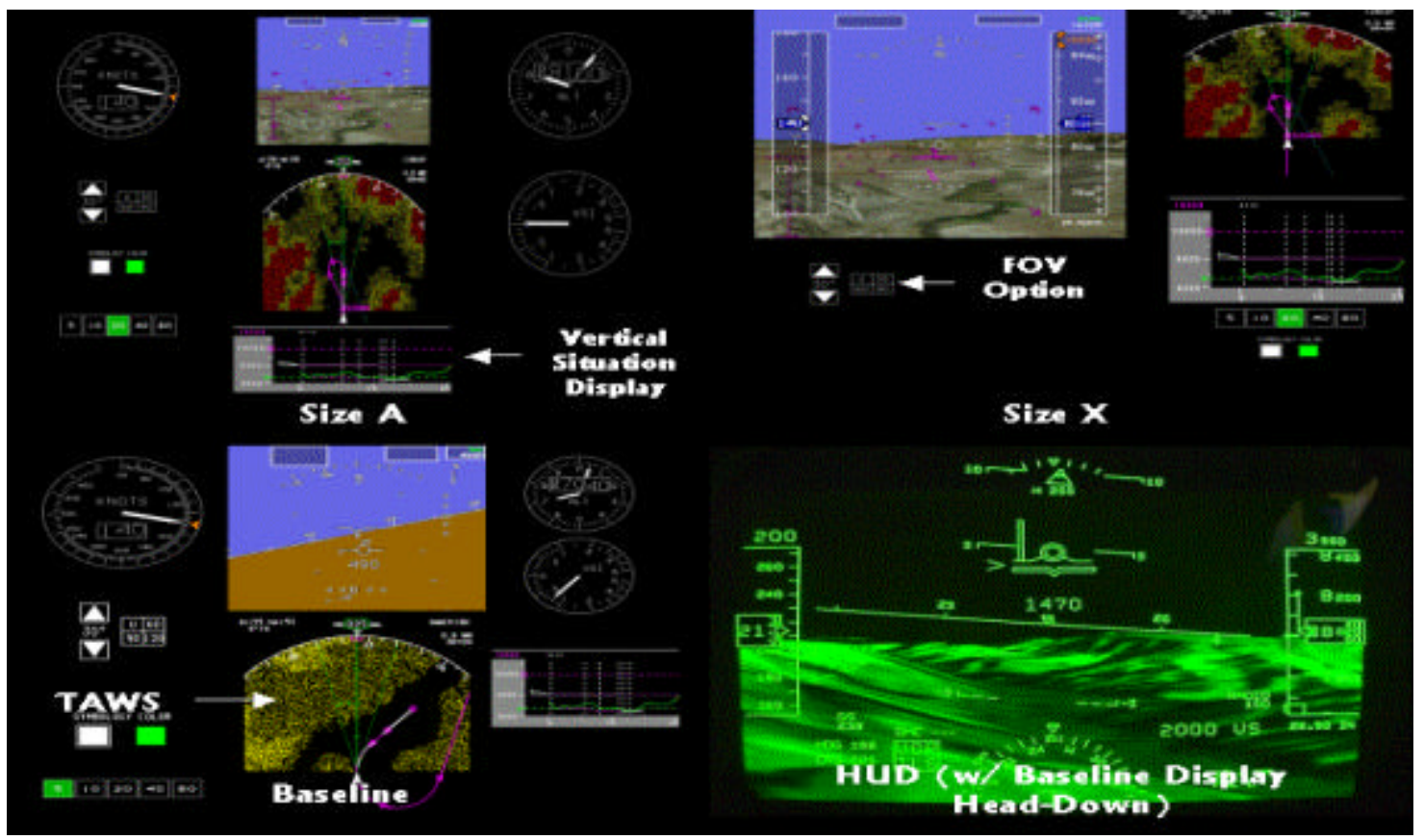

Figure 5. Display Concepts For Experiment II 


\section{Display Conditions}

The display concepts were: (a) baseline EFIS 757 display, (b) size A (5.25" x 5.25") display with SVS, (c) size X display size (8"x10") with SVS, and (d) HUD enhanced with SVS. For all display presentations, the ND with TAWS and VSD was a size B (4" x 6"). For the HUD, the HDD was the baseline (EADI, TAWS and ND) concept (Figure 5). The order of display presentation was randomized across evaluation pilots. Only the baseline and photo-realistic SVS concepts were flown during the CFIT scenario because past research (Prinzel et al., 2002) reported no significant differences between generic and photo-realistic terrain texturing for terrain awareness.

\section{Pilot Performance}

Results

Nominal Go-Around Performance. Pilots were instructed to maintain a speed-on-pitch target of 140 knots on climb-out during go-around and to minimize lateral path deviation. An ANOVA found a significant main effect for Display Type for RMS lateral performance $(\mathrm{F}(3,15)=$ 22.612, $\mathrm{p}<.01)$. RMS error was significantly greater for Baseline (504ft.) than for HUD (91 ft.), Size A (107 ft.), and Size X (142 ft.). No significant effect was found for commanded speed deviation, $\underline{p}<.05$, although RMS flight director pitch commands were significantly greater for Baseline (3.37) than for HUD (1.29), Size A (1.73), and Size X (1.69) suggesting that pilots had to make more pitch adjustments to maintain commanded speed with the Baseline display than with SVS concepts.

CFIT Event. For the CFIT scenario, 12 of the 16 test subjects flew the CFIT scenario with a SVS enhanced PFD or HUD and 4 pilots flew the Baseline display. All 12 SVS pilots noticed and avoided the CFIT. On average, pilots with a SVS display noticed the potential CFIT 53.6 seconds before impact with the terrain, but all four Baseline pilots $(100 \%)$ had a CFIT event. Three of the 4 pilots impacted the terrain while one passed within 58 feet of a mountain peak (topped trees on mountain). Even though the baseline concept had a Radio Magnetic Indicator (RMI), TAWS and VSD enhanced ND, none of the Baseline pilots were aware until after the CFIT event had occurred (Figure 6). It should also be noted that 2 CFIT events occurred during nominal trials while pilots flew the Baseline display and collided with the ground during the turnto-final to runway 07.
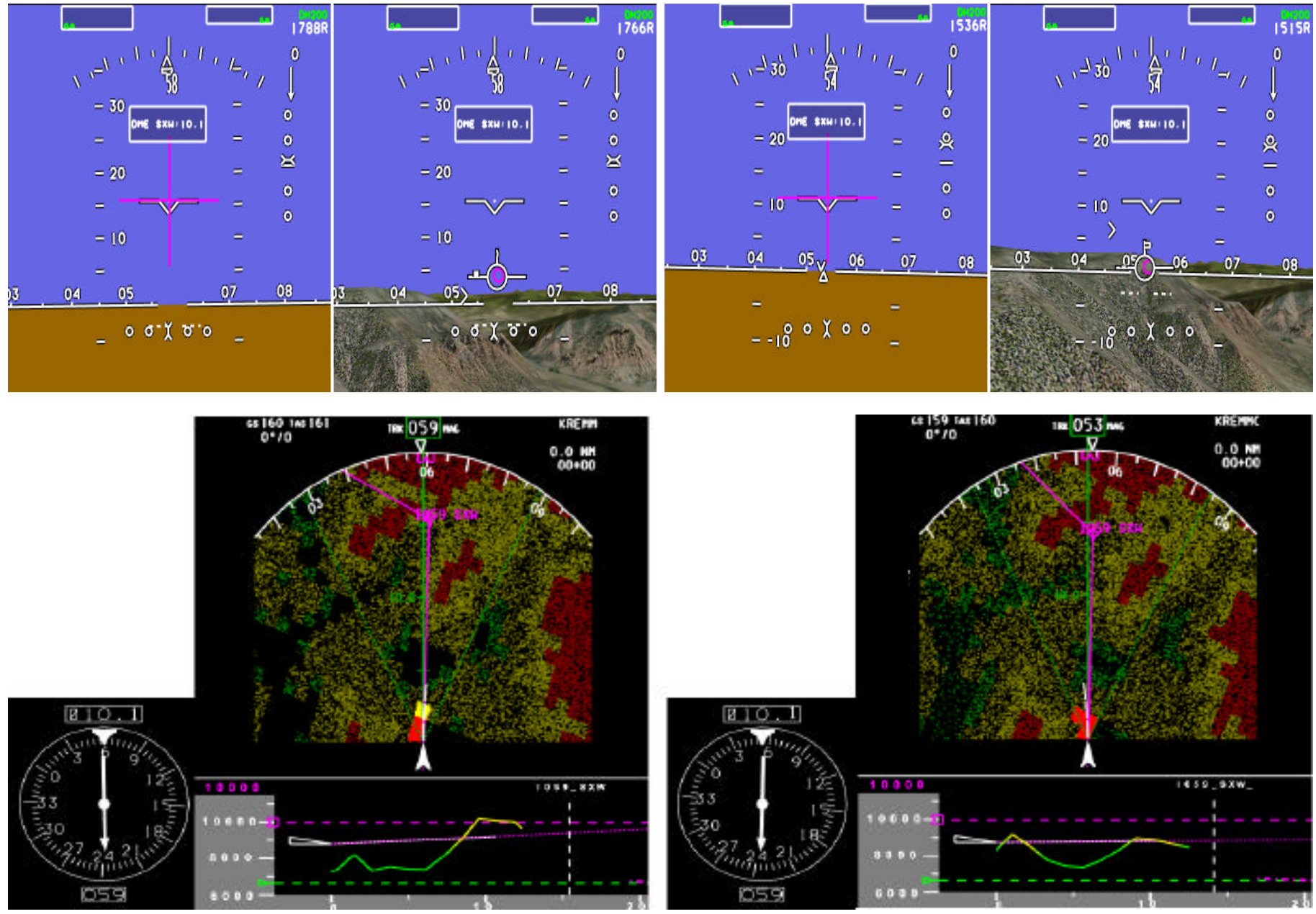

Figure 6. Display Presentation During Nominal (Left) and CFIT Scenarios (Right)

Pilot Workload

Modified CH. Display type $(\mathrm{F}(3,15)=10.286$, $\mathrm{p}<.001)$ was found to be significant for the Modified Cooper-Harper ratings. Post Hoc tests evinced two unique subsets with Size X (1.25) and HUD (1.25) being rated significantly lower in workload than Size A (2.75) and Baseline (3.75). The ratings for Baseline concept (moderately high mental workload) indicate that the mental work level was not acceptable during the go-around, CFIT scenario. 
Post-Run Questionnaire. An ANOVA for "ease and precision" (0 to 6), however, was not found to be significant $(\mathrm{F}(3,15)=2.419$, $\mathrm{p}<$. 117). Pilots slightly agreed that the Baseline concept (4.0) could be flown with ease and precision in contrast to moderately agree ratings for Size A (4.75) and HUD (5.0) and strongly agree ratings for Size X (5.50).

Semi-Structure Interview. Display type was, however, found to be significant for workload during the go-around CFIT event, F $(3,15)=$ 93.651, $\mathrm{p}<.0001$. On a scale of 0 to 10 , pilots rated the Baseline display (3.62) significantly higher in workload than Size A (6.87), HUD (7.13), and Size X (8.09). A rating of 3.62 for Baseline reflects a "somewhat high" workload rating compared to "somewhat low" (Size A; HUD) and "very low" workload ratings (Size X).

\section{Pilot Situation Awareness}

Post-Run Questionnaire. Display type was not found to be significant for terrain awareness ( $\underline{p}>$.05). Mean ratings for both questions, however, indicate that the HUD and Size X display concepts provided better terrain awareness and aircraft position that Size A and Baseline. An ANOVA did not find that these results for workload or terrain awareness were due to an inability of the pilot to interpret the display information $(\mathrm{F}(3,15)=0.560, \mathrm{p}<.651)$.

Semi-Structured Interview. An ANOVA reported a significant result for Display Type for situation awareness, F $(3,15)=76.605$, $\underline{\mathrm{p}}<$ .0001. Pilots rated situation awareness for Baseline to be "very low" (2.50) compared to "somewhat high" ratings for Size A (7.06) and HUD (7.09) and "very high" SA for Size X (8.75).

SA-SWORD. Pilots were asked to complete a SA-SWORD for situation awareness comparison across the display conditions (including generic texture versions). The definition of SA was defined as: "The pilot's awareness and understanding of all factors that will contribute to the safe flying of their aircraft under normal and non-normal conditions." Pilots were asked to make these ratings based on their operational experience as commercial pilots. An ANOVA found a significant effect for Display Type, F $(6,90)=14.443$, $\mathrm{p}<.001)$. Size X was found to provide significantly higher SA than all other Display Types, and Baseline was rated lowest in SA for all Display Types although was not found to be significant from Size A Generic Texture.

\section{DISCUSSION}

"In art a picture may be worth a thousand words, but with synthetic vision a picture may be worth a thousand lives." --- Captain, United Airlines

The problem of reduced visibility challenges aviation goals to reduce the accident rate and improve operational capacity (FAA, 2001; NASA, 2001). The approach of synthetic vision is to solve the problem through the presentation of how the outside world would look to the pilot if vision were not restricted. The results of these two experiments demonstrate the efficacy of SVS to significantly reduce the CFIT accident rate for both GA and commercial operations not possible with current systems. Although systems such as Terrain Awareness Warning Systems are steps in the right direction and have significantly improved safety, the solution treats the symptoms and not the cause; they are still "warn-act" systems (Moroze \& Snow, 1999). Synthetic vision instead allows for proactive prevention of visibility-induced accidents while also increasing the capability to make approaches in weather conditions and airports not currently available for low-visibility operations.

The concept described here represents only the database and display concepts and not the total synthetic vision system, which will include synthetic vision navigation displays; runway incursion prevention technology; database integrity monitoring equipment; enhanced vision sensors; taxi navigation displays; and advanced communication, navigation, and surveillance. These technologies represent a comprehensive solution to restricted visibility that will be researched in near-term NASA flight tests to be conducted during the summer of 2003 . The research will focus on the integration of runway incursion prevention technologies, surface map displays, integrity monitoring, enhanced sensors, and enhanced synthetic vision displays. Together, such a synthetic vision system may considerably help meet national aeronautic goals to "reduce the fatal accident rate by a factor of 5" and to "double the capacity of the aviation system" both with 10 years (NASA, 2001).

\section{REFERENCES}

Federal Aviation Administration (2001). FAA Strategic Plan. Washington, D.C.: FAA

Glabb, L.J., \& Takalu, M.A. (2002). Preliminary effect of synthetic vision systems displays to reduce low-visibility loss of control and controlled flight into terrain accidents. SAE Technical Paper 2002-01-1550. Warrendale, PA: Society of Automotive Engineers.

Hughes, M.F., \& Takallu, M.A. (2002). Terrain Portrayal for Head-Down Displays Ex neriment. Proceedings of International Advanced Aviation Technologies Conference, G-1 - G-12.

Moroze, M.L., \& Snow, M.P. (1999). Causes and remedies of controlled flight into terrain (CFIT) in military and civil aviation. Proceedings of the $10^{\text {th }}$ International Symposium on Aviation Psychology. Columbus, OH: Ohio State University.

National Aeronautics and Space Administration (2001). Aerospace Technology Enterprise. Washington, D.C.: NASA.

Prinzel, L.J., Kramer, L.J., Comstock, J.R., Bailey, R.E., Hughes, M.F., \& Parrish, R.V. (2002). NASA synthetic vision EGE flight test. Proceedings of the Annual Human Factors and Ergonomics Meeting, 46, 135-139.

Prinzel, L.J., Comstock, J.R., Glaab, L.J., Kramer, L.J., Arthur, J.J., \& Barry, J.S. (in press). The efficacy of head-down and head-up synthetic vision display concepts for retro- and forward-fit of commercial aircraft. International Journal of Aviation Psychology.

Williams, D., Waller, M., Koelling, J., Burdette, D., Doyle, T., Capron, W., Barry, J., \& Gifford, R. (2001). Concept of operations for commercial and business aircraft synthetic vision systems. NASA Langley Research Center: NASA Technical Memorandum TM-2001211058 . 